\title{
Adjusting the Frequency of Automated Phosphorus Measurements to Environmental Conditions
}

\author{
Hermann Klug ${ }^{1}$, Alexander Kmoch ${ }^{2}$ and Steffen Reichel ${ }^{1}$ \\ ${ }^{1}$ Z_GIS, University of Salzburg, Austria ·hermann.klug@sbg.ac.at \\ ${ }^{2}$ Geoinformatics Research Centre, Auckland University of Technology, New Zealand
}

Full paper double blind review

\begin{abstract}
Extreme weather events are likely to increase in the future, and thus damage to the environment and infrastructure will likely increase during this time, too. To adapt to these weather impacts, forecasting, now-casting, and in situ monitoring installations have increased during the last years. Even though monitoring stations deliver frequent measurements in realtime, a dynamic implementation of measurement frequencies, adapted to certain environmental conditions, are rarely implemented. Within this paper we provide a framework where low frequency phosphorus measurements in the Mondsee catchment can be adapted to high frequency measurements during storm events. When heavy rainfall is observed, a threshold event triggers a reconfiguration task for the phosphorus measurement device, using asynchronous, push-based communication. A Sensor Planning Service commits such a request into the wireless sensor network, and updates the measurement frequency of the target nodes to enable nutrient peak flow estimation during storm events. This setup introduces the possibility of measurements in flooded areas without using traditional (manual) sampling methods, and we expect to obtain a better understanding of discharge to phosphorus runoff relations.
\end{abstract}

\section{Introduction}

Storm events will likely increase in Europe (CZIGÁNY et al. 2011) and the European Alps (DOBLER et al. 2013). For the pre-Alpine Lake District around the provincial capital city Salzburg, an increase of $20 \%$ is expected for extreme precipitation events in the summer period between 2007-2050 (HOFSTÄTTER et al. 2010). Two flooding impacts from those kinds of events were recently visible in the Mondsee catchment, lasting a few days after June 2nd, 2013 and October 23rd, 2014.

During these storm events, the sediment load of the rivers and lakes in the Mondsee catchment increased, as did the attached particle bound phosphorus nutrients (SWIERCZYNSKI et al. 2013). STRAUSS \& STAUDINGER (2007) estimated that during an earlier flood in August 2002, almost half of the total annual phosphorus load from the River Fuschler Ache entered Lake Mondsee. The increase of particle input into the lake (considerably during the 2013 flooding event) was confirmed by MUELLER et al. (2013). Even during meteorologically 'normal' years, up to $80 \%$ of the total phosphorus load of Lake Mondsee originated from single events in the snow melting period from February to March, and occasional (2-3 times 
per year) heavy rainfall ( $>50 \mathrm{~mm} \mathrm{~d}^{-1}$ ) in summer (STRAUSS \& STAUDINGER 2007). The importance of the frequency and intensity of rainfall on the concentration of soluble phosphorus in runoff is also evident from studies in other areas (ANN et al. 1999, JORDAN et al. 2005a, JORDAN et al. 2005b). This inter-annual frequency of changes has been neglected in a spatially explicit modelling approach, conducted by KLUG \& ZEIL (2008). Thus, with an estimate of $12 \mathrm{t}$ per year, STRAUSS \& STAUDINGER (2007) underestimated the total annual phosphorus loads into the Mondsee, while KLUG \& ZEIL (2008) overestimated the phosphorus loads with $17 \mathrm{t}$ per year in their modelling approach.

Phosphorus discharge during extreme events has not been continuously recorded in the Mondsee catchment. This was impossible due to wide inundation areas, impeding access to phosphorus samplers. Furthermore, the sampler could only take a small number of field samples to be analysed in the laboratory soon after sample taking, to avoid re-suspension of phosphorus to particles. This would disable a clear distinction between Orthophosphate $\left(\mathrm{PO}_{4}{ }^{3-}\right)$, total phosphorus loads, and plant available phosphorus (soluble reactive phosphorus, SRP), causing the eutrophication in the oligo-mesotrophic Lake Mondsee with around $10 \mathrm{mg} \mathrm{P} \cdot \mathrm{m}^{-3}$ (ACHLEITNER et al. 2007).

To enable a place independent sampling and real-time analysis we use the wireless sensor network setup, as explained in KLUG et al. (2014). In this manuscript we detail the process framework from steering the phosphorus measurement device Systea WIZ (Water Insitu AnalyZzer), and follow the approach of KLUG \& KMOCH (2015) for the landscape lab configuration. Bidirectional sensor uplink methods are proposed by HUNKELER et al. (2013). They present a sensor network architecture that is controlled centrally. Vertically it integrates a Wireless Sensor Network's (WSN) network stack with the publish/subscribe messaging middleware MQTT-S (Message Queue Telemetry Transport). Also CHEN et al. (2013) discuss MQTT as a suitable bi-directional transport protocol for wireless sensor networks following the message queue pattern.

The Open Geospatial Consortium (OGC) provides a set of standards that enable such a desired architecture. For the domain of linking sensor networks into a service oriented architecture, the OGC defines a set of service interfaces and data encodings, in particular through the Sensor Web Enablement initiative (SWE) (BOTTS et al. 2008). "The Sensor Web Enablement (SWE) standards of the Open Geospatial Consortium (OGC) comprise the only truly open international standards suite that provides a comprehensive platform for publishing, discovering, assessing, accessing, and using sensors and sensor systems of all kinds" . Message encoding within SWE services is defined in the specifications for OWS Commons (OGC Web Service Commons), SWE Common Data Model Encoding (OGC 2011a), Observations \& Measurements (O\&M) (OGC 2011b), and SensorML (OGC 2007). The sensor web standards have been exhaustively described in (BRÖRING et al. 2011). O\&M is a generic flexible application schema for the encoding of observation data and time-series, whereas WaterML2.0 is an encoding that extends O\&M and has been specifically created for hydro-climate observations and time-series. Sensor descriptions, configurations, and capabilities can be expressed in SensorML. Main interfaces for data transmission and interaction with sensors and actors are the Sensor Observation Service (SOS) and the Sensor Planning Service (SPS) (OGC 2011c).

\footnotetext{
1 OGC Sensor Web Enablement (SWE) Homepage: http://www.ogcnetwork.net/swe
} 
Within this manuscript, we explore whether the OGC SWE semantics for sensor descriptions and observations (SOS), sensor configuration, and planning (SPS) with the open MQTT protocol can be interlinked to enable a flexible bidirectional eventing and tasking infrastructure for the wireless sensor network.

\section{Material and Methods}

\subsection{The Mondsee Catchment}

The $248 \mathrm{~km}^{2}$ Mondsee catchment is endowed in the pre-alpine Lake District located to the east of the Austrian provincial city Salzburg (KLUG 2010). Lake Mondsee and its catchment belong to the Austrian Long-Term Ecosystem Research Network (LTER-AT), and is subject of studies related to climate change and its effects to the terrestrial and aquatic ecosystem.

\subsection{The Landscape Lab}

\subsubsection{General Setup}

While Lake Mondsee itself is an aquatic study subject, this paper focusses on a terrestrial study area in the $6 \mathrm{~km}^{2}$ sub-catchment Koppl, located in the west of the Mondsee catchment. The geoscientific landscape lab comprises a WSN, with a main station at the 'Meindlbauer', conducting comprehensive measurements of meteorological, hydrological and pedological parameters (KLUG et al. 2014). For the wireless data transmission within the WSN XBee-PRO modules from the Digi Company implementing the ZigBee IEEE 802.15.4 protocol are used (BORMANN et al. 2013, ABRAHAM \& LI 2014). The main station receives continuous sensor measurements from eight substations, and acts as the gateway to the online SOS and SPS services by providing the communication channel from the local sensor network to the web-enabled data management infrastructure. Station two, 'Plainfelder Bach', measures discharge and phosphorus loads at the confluence of 'Koppler Bach' and 'Plainfelder Bach', and is of interest here. This station comprises a Sontek IQ-Plus (discharge), a Water Insitu AnalyZer, and additional technical infrastructure to support energy supply. Because the station 'Plainfelder Bach' is located in a catchment valley, it cannot establish a reliable wireless ZigBee connection with the main station. Therefore, station 'Plainfelder Bach' utilises a Raspberry PI as a gateway, with a mobile Internet connection for data distribution, and can be considered a second independent WSN. The station 'Plainfelder Bach' is also connected to the joint web-enabled data management infrastructure.

\subsubsection{Water Insitu AnalyZer}

The Systea WIZ analyses total phosphorus (TP, detection range: 0-1 mg/L P [0 - $1000 \mathrm{ppb}$ $\mathrm{P}])$, total organic available phosphorus $\left(\mathrm{P}_{\text {org }}\right.$; detection range: $\left.0-1 \mathrm{mg} / \mathrm{L} \mathrm{P}[0-1000 \mathrm{ppb} \mathrm{P}]\right)$, and orthophosphate $\left(\mathrm{PO}_{4}{ }^{3-}\right.$; detection range: $\left.0-0.500 \mathrm{mg} / \mathrm{L} \mathrm{P}-\mathrm{PO}_{4}[0-1000 \mathrm{ppb}]\right)$. It is an innovative in-situ probe, using the Systea patented micro Loop Flow Analysis ( $\mu$ LFA) technique, combined with a multi-parametric sequential reactor, to measure phosphorus compounds in surface water. Chemical reagents are located in a hand-held canister on top of the IP68 protected field device. 
The WIZ is a stand-alone working field laboratory, powered at $12 \mathrm{Vdc}$, consuming $0.3 \mathrm{~A}$ $(3 \mathrm{~W})$ in stand-by mode and $1.6 \mathrm{~A}(6 \mathrm{~W})$ in average during analysis. Samples are analysed according to the molybdenum blue method, after MURPHY \& RILEY (1962), measuring intensity at $880 \mathrm{~nm}$ spectrum, and providing analysis results after 512 seconds (temperature $20^{\circ} \mathrm{C}$ ) for $\mathrm{PO}_{4}{ }^{3-}, 1112$ seconds for $\mathrm{P}_{\text {org, }}$ and 2815 seconds for TP at an RS-232 standard serial port. We use the serial protocol commands replacing the provided software to enable an operation based on environmental conditions, as described below.

Lowest sample frequency is approximately one hour for TP, while the number of measurements is dependent on availability and consumption of chemicals. Reagents consumption is reduced to about 30-60 microliters of each reagent per single analysis. The reagents in the hand-held canister are designed to contain up to $300 \mathrm{ml}$ of reagents and calibrant solution, to ensure around 400 automatic field analyses. However, the chemical substances should be changed every three months to guarantee high quality measurements.

\subsubsection{Wireless Sensor Network Infrastructure}

The station 'Plainfelder Bach' as an independent WSN follows the design principles of the 'Meindlbauer' main station. It consists of an autarchic energy supply using a 300Wp solar panel and a $300 \mathrm{~W} 12 \mathrm{~V}$ wind generator, connected to a $260 \mathrm{Ah} 12 \mathrm{~V}$ battery using charge regulators. The central field computer (a Raspberry PI gateway) is equipped with a 3G / GPRS modem and a SIM card, and receives the measurements from the discharge and phosphorus sensors. Both devices are connected to the field computer via an RS232 connection. Additionally, there is a weather station installed near station 'Plainfelder Bach', communicating wirelessly with the Raspberry PI field computer via the ZigBee connection. The field computers are connected to the internet-based sensor network management framework via an MQTT communication protocol. The collected measurements are regularly inserted into an SOS. The field computers can receive sensor planning or reconfiguration commands via SPS tasking messages. Both WSNs are connected to the same MQTT queue, which links them with the SOS and SPS services.

\subsection{Sensor Network Management Framework}

The data and sensor management is based on distributed services that are connected via the Internet, and exchange messages based on SWE semantics. External access is provided to the measurement data via SOS, or notifications via emails, SMS, or mobile applications. We use SWE semantics for sensor descriptions and observations (SensorML, O\&M), sensor configuration and planning (SPS tasking), in combination with the open MQTT protocol. MQTT is a publish/subscribe message passing system, developed for inherently unreliable networks and the Internet of Things (IoT) applications. We employ MQTT as event brokering and message passing, as implied by the SWE Service Model to connect the WSNs in the field with the SWE services framework in the Internet. Actual addressing and data transmission is done by a topic string and an arbitrary payload. The topic string and the payload need to reflect the necessary SWE standard semantics to allow for a seamless and lossless bi-directional, multi-lateral communication between the WSN. The proposed architecture is illustrated in Fig. 1. 
- MQTT channel

- Connecting internet and wireless sensor network

- Transmits messages based on SWE semantics



Fig. 1: Bi-directional communication between management services and sensor network

A $52^{\circ}$ North SOS server is used to store measurements from the WSNs 'Meindlbauer' (station one) and 'Plainfelder Bach' (station two) in the data formats Observations \& Measurements and WaterML2.0. Observation messages are typically sent from the field computer, and are inserted via an SOS_InsertObservation operation. Data can be requested via an SOS_GetObservation operation, and the result data will be encoded in O\&M or WaterML2 for subsequent data processing algorithms. The configuration, components, and characteristics of the sensors at the field stations are described in SensorML in the SOS, and can be retrieved via the SOS_DescribeSensor operation.

\subsection{Sensor Eventing and Tasking}

Rainfall is measured continuously at the distributed rain gauges of the field stations in the Koppl catchment, and is subsequently transmitted via the gateways over the queue to be stored in the SOS. A still to be defined amount of rainfall per time should increase the low frequent phosphorus analyses to high frequent samples during extreme events. When the snow season is ending and the spring freshet approaches, the same procedure applies as when snow water equivalents measurements decrease at the Snow Pack Analyser, and discharge increases at station 'Plainfelder Bach'. The framework of this sensor logic is integrated in an SPS, and automatically relates the frequency of environmental measurements to environmental conditions.

A monitoring process is configured with the desired threshold values for observed rainfall, as well as the stream flow at the defined measurement stations. The process is run within the management framework, and filters the latest rainfall, snow water equivalents, and flow observations from the field stations, and evaluates them against the configured thresholds. If values rise above the defined limits an event is raised, which will be acted upon by two subsequent processes. The SPS will issue a tasking message to be sent via the MQTT channel. The field computers read the message based on topic string and payload. The station 
'Plainfelder Bach' in this case reacts to messages regarding reconfiguration of the phosphorus sensor, and translates the reconfiguration towards the phosphorus station. Additionally, the event will trigger notifications to inform configured recipients, i.e. email, SMS.

The phosphorus device is reconfigured by the field computer to the new sampling setup (i.e. increase measurement frequency and type of P-analysis) and will continue with this new setup until a new notification sets it back to standard low frequency sampling. When the field computer receives the acknowledgement from the phosphorus station that the reconfiguration has been accepted, it updates the descriptive SensorML in the SOS. In this way, the SOS_DescribeSensor displays the latest sensor configuration of the network.

Analogously, once the observed rainfall and flow measurements decrease below the limits, an antagonistic process will send a reconfiguration message to redeem 'flood' conditions, i.e. task to decrease measurement frequencies again.

\section{Results}

The implemented infrastructure is explained in Fig. 2. Both WSNs regularly push environment data into the SOS via the shared MQTT queue $(1,2)$. A regular time based process retrieves the latest rainfall observations (3). The filtering process accumulates over a given time period, and triggers a reconfiguration event in the SPS when a threshold is met (4). The SPS issues a tasking message on the message queue, which the WSN field computer consumes and then reconfigures (6) the phosphorus measuring device. The field computer updates the SensorML to reflect the configuration change (7). Simultaneously, the time based process (3) notifies decision makers via e-mail or SMS (5) about the rainfall event and the initiated a configuration change.

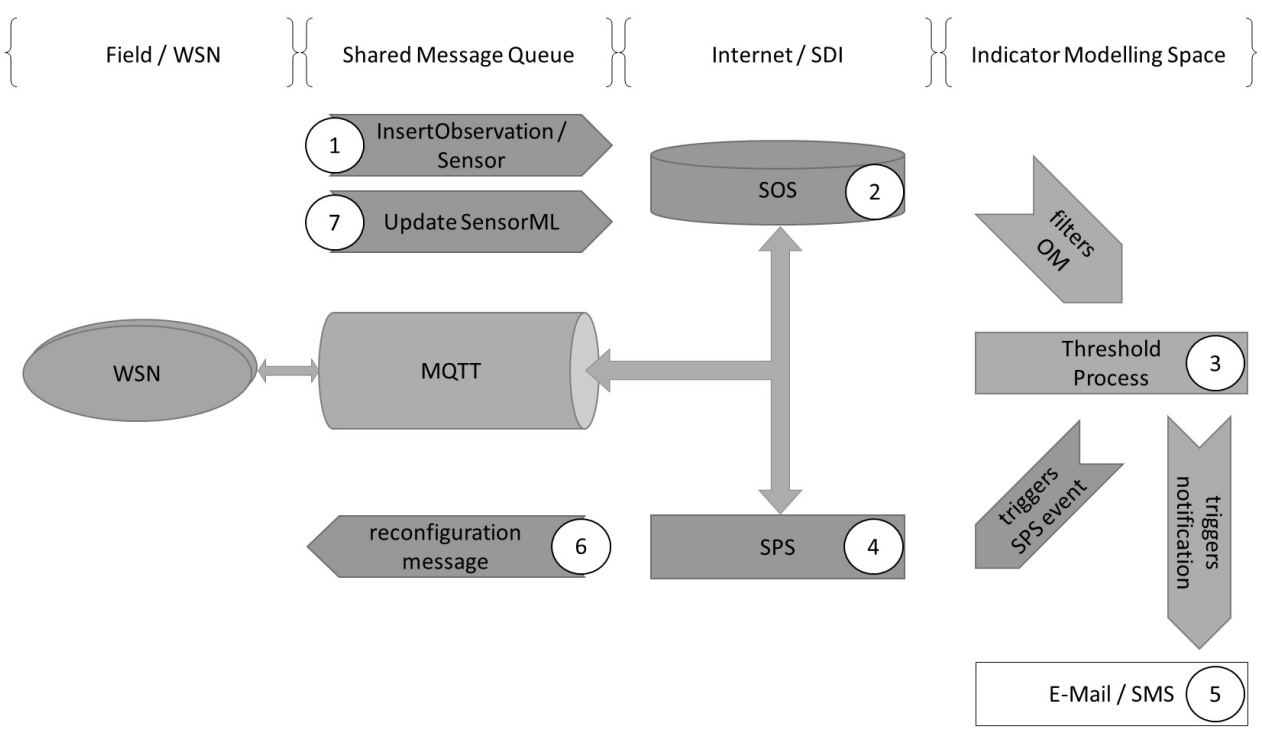

Fig. 2: Message passing infrastructure 
The analogous process restarts when a lower threshold is met, or if the observed measurements fall below the actual threshold that is configured to indicate high rainfall. A reconfiguration message to the phosphorus measuring device will be issued, and induce a lower sampling frequency. Then, as an acknowledgement, the SensorML in the SOS will also be updated to reflect a change of configuration in the online data management infrastructure. Furthermore, a notification is sent to stakeholders that the state of the catchment and the measurements are back to normal.

\section{Discussion}

STRAUSS \& STAUDINGER (2007) based their total annual phosphorus load on samples during high flow and low flow conditions, and related water discharge and phosphorus runoff for the estimation of the annual load. While phosphorus discharge during spring freshet matches well, their figures show an underrepresentation in phosphorus loads during storm events. While STRAUSS \& STAUDINGER (2007) seemed to underestimate the total annual phosphorus loads, KLUG \& ZEIL (2008) seemed to overestimate the loads with their modelling approach. This overestimation results from averaging environmental conditions, not taking into account short-term extreme events at all. Since storm events play an important role in the phosphorus runoff (JORDAN et al. 2005b), but no phosphorus runoff-discharge relationship was available for the Mondsee catchment, we set up a framework for better accounting phosphorus loads during storm events.

With our setup, we were able to test the described procedure. With the IP68 protected device we can ensure to measure at any place, especially at those places where 'normal' samplers fail to measure due to the impact of the flood. An energy autarchic setup using solar radiation and wind enabled us an independent place for measurements. With the minimum use of chemical substances and the sealed bags of reagents we could ensure a reduced return time to the observation place, and could ensure receiving phosphorus values immediately after the field-laboratory procedure. Extra efforts for collecting samples and laboratory analysis shortly after sample taking can be avoided. We have not tested the setup for a longer time period, and have not exhaustedly tested the setup during extreme events, thus we cannot provide an indication of maintenance work needed yet. However, a very recent study from BowES et al. (2015) with a similar device shows a continuous field observation from January 2010 to December 2011 and would support the hypothesis that long term operation even during extreme events is possible.

Finding an appropriate threshold value for device configuration seems more challenging than expected, since many parameters control the pattern and processes during low flow events with $2 \mathrm{~m}^{3} / \mathrm{s}$ and $0,01 \mathrm{mg} \mathrm{P} / \mathrm{L}(10 \mathrm{ppb})$ and high discharges with $50 \mathrm{~m}^{3} / \mathrm{s}$ and around $0,4 \mathrm{mg} \mathrm{P} / \mathrm{L}$ (400 ppb) discharge. Rainfall alone can trigger the device without a significant increase of discharge, since infiltration capacities might be high and runoff delayed. Also, changes in snow water equivalents strongly depend on frozen (sub-)surfaces and/or high/ low water infiltration capacities into the soil. The most reliable trigger in our tests was discharge to be related to phosphorus runoff. This would enable the requested relationship for better accounting for the annual phosphorus input into Lake Mondsee, but would not help to locate rainfall-runoff from certain locations in the catchment. Thus, without the discharge measurement, a proper relationship cannot be established yet. 
The real-time monitoring system and web-based information system are designed for use by decision makers. With the leveraging of the established OGC Sensor Web Enablement standards, and the reliability, performance, efficiency, and robustness of the MQTT protocol, Wireless Sensor Networks can be linked directly to ubiquitous SDI and web services architectures for online data access and sensor configuration. While the technical infrastructure has been tested reliable, the continuous field measurements have not been cross-validated with laboratory analyses yet. However, we compared the measurement of the Systea WIZ with calibration reagents in the laboratory, and achieved accuracies close to $100 \%$ accuracy for peak flow (500 ppb) and for low flow conditions (25 ppb). These accuracies have not been detailed here, and depend on the type of phosphorus and the level of phosphorus to be analysed.

After an increase of the frequency of environmental measurements, the re-scheduling from higher to lower frequencies can only be reliably done by the discharge measurements. The lag time of water runoff after the precipitation stopped, cannot yet be estimated properly. Further experiences need to broaden the understanding of the hydro-meteorological spatiotemporal processes and functions in the particular catchment.

\section{Conclusion and Outlook}

We enabled a non-real-time working phosphorus monitoring device to provide frequently updated phosphorus measurements from the field, without approaching the device, or being required to manually take water samples in the field. Based on an automated setup of a standard compliant infrastructure, we could trigger measurement frequency and type of phosphorus analysis from our office computers. Additionally, environmental measurements and a defined ruleset enable the automatic tasking of higher frequency measurements during extreme events. This is expected to increase an understanding of discharge to phosphorus runoff relationships, and is expected to increase our knowledge of time-dependent impacts on the lake ecosystem to be analysed with a FlowCam installed in Lake Mondsee in future.

We could show that the implemented message passing infrastructure is effective to allow for high level OGC SWE semantics on a low-level message transport protocol. The convergence of SDI, sensor web, and message passing can be seen as an important step towards a robust and efficient implementation of the Internet of Things.

\section{Acknowledgements}

We would like to acknowledge the Doctoral College GIScience (DK W 1237-N23) of the University of Salzburg for providing support based on funding received from the Austrian National Science Fund (FWF). We also would like to acknowledge the on-going collaboration with farmers providing access to their land and space for sensor instalments. We also thank Nicola Hüsing for providing access to the chemical laboratory and Systea staff members for continuous support on their device. 


\section{References}

Abraham, S. \& Li, X. (2014), A Cost-effective Wireless Sensor Network System for Indoor Air Quality Monitoring Applications. Procedia Computer Science, 34(0), 165-171.

Achleitner, D., Gassner, H. \& JAGSCH, A. (2007), Die limnologische Langzeitentwicklung des Mondsees und Irrsees. In: STRAuSS, P. (Hrsg.), Ausgewählte Ergebnisse des INTERREG IIIA-Projektes „SeenLandWirtschaft“. Bundesamt für Wasserwirtschaft, Petzenkirchen, 26, 3-17.

ANN, Y., REDDY, K. R, \& DELFINO, J. J. (1999), Influence of chemical amendments on phosphorus immobilization in soils from a constructed wetland. Ecological Engineering, 14 (1-2), 157-167.

Bormann, K. J., Westra, S., Evans, J. P. \& McCabe, M. F. (2013), Spatial and temporal variability in seasonal snow density. Journal of Hydrology, 484 (0), 63-73.

Botts, M., Percivall, G., Reed, C. \& Davidson, J. (2008), OGC® Sensor Web Enablement: Overview and High Level Architecture. In: NitTEL, S., LABRINIDIS, A. \& STEFAnIDIS, A. (Eds.), GeoSensor Networks. Springer, Berlin/Heidelberg. 4540, 175 190.

Bowes, M. J., Jarvie, H. P., Halliday, S. J., Skeffington, R. A., Wade, A. J., Loewenthal, M., Gozzard, E., Newman, J. R. \& Palmer-Felgate, E. J. (2015), Characterising phosphorus and nitrate inputs to a rural river using high-frequency concentration flow relationships. Science of The Total Environment, 511 (0), 608-620.

Bröring, A., Echterhoff, J., Jirka, S., Simonis, I., Everding, T., Stasch, C., Liang, S. \& Lemmens, R. (2011), New Generation Sensor Web Enablement. Sensors, 11 (3), 2652-2699.

Chen, J., DÍAZ, M., Rubio, B. \& Troya, J. M. (2013), PS-QUASAR: A publish/subscribe QoS aware middleware for Wireless Sensor and Actor Networks. Journal of Systems and Software, 86 (6), 1650-1662.

Czigány, S., Pirkhoffer, E., NAgyváradi, L., Hegedüs, P. \& Geresdi, I. (2011), Rapid screening of flash flood-affected watersheds in Hungary. Zeitschrift für Geomorphologie, Supplementbände 55 (1), 1-13.

Dobler, C., BÜRGER, G. \& STÖTTER, J. (2013), Simulating future precipitation extremes in a complex Alpine catchment. Nat. Hazards Earth Syst. Sci., 13, 263-277.

Hofstätter, M., Matulla, C., WANG, J. \& WAGNer, S. (2010), PRISK-CHANGE Veränderung des Risikos extremer Niederschlagsereignisse als Folge des Klimawandels. Projektbericht, Zentralanstalt für Meteorologie und Geodynamik (ZAMG).

Hunkeler, U., Lombriser, C., Truong, H. L. \& Weiss, B. (2013), A case for centrally controlled wireless sensor networks. Comput. Netw., 57 (6), 1425-1442.

Jordan, P., ARnSCheidT, J., MCGrogan, H. \& MCCORMicK, S. (2005a), High-resolution phosphorus transfers at the catchment scale: the hidden importance of non-storm transfers. Hydrol. Earth Syst. Sci., 9 (6), 685-691.

Jordan, P., Menary, W., Daly, K., Kiely, G., Morgan, G., Byrne, P. \& Moles, R. (2005b), Patterns and processes of phosphorus transfer from Irish grassland soils to rivers - integration of laboratory and catchment studies. Journal of Hydrology, 304, 20-34.

KLUG, H. (2010), Application of a vision in the Lake District of Salzburg. Journal Futures, 42 (7), 668-681. 
Klug, H., Kmoch, A. \& Juhasz, C. (2014), Drahtlose Echtzeitanalysen von Phosphorausträgen bei klimatischen Extremereignissen. In: STROBL, J., BLASCHKE, T., GRIESEBNER, G. \& ZAGEL, B. (Hrsg.), Angewandte Geoinformatik 2014. Beiträge zum 26. AGIT-Symposium in Salzburg. Wichmann, Berlin/Offenbach, 548-557.

KLUG, H. \& KMOCH, A. (2015), Operationalizing environmental indicators for real time multi-purpose decision making and action support. Journal Ecological Modelling, 295, 66-74.

KLUG, H. \& ZeIL, P. (2008), Spatially Explicit Modelling of Phosphorus Emissions. Geoinformatics, 8 (11), 32-35.

Mueller, P., Thoss, H., KaempF, L. \& Güntner, A. (2013), A Buoy for Continuous Monitoring of Suspended Sediment Dynamics. Sensors, 13 (10), 13779-13801.

MurPhy, J. \& RiLEY, J. P. (1962), A modified single solution method for the determination of phosphate in natural waters. Analytica Chimica Acta, 27 (0), 31-36.

OGC (2007), Sensor Model Language (SensorML) Implementation Specification. 1.0.0. http://www.opengeospatial.org/standards/sensorml, The Open Geospatial Consortium (OGC).

OGC (2011a), OGC SWE Common Data Model Encoding Standard, v2.0. SWE 2.0. http://www.opengeospatial.org/standards/is, The Open Geospatial Consortium (OGC).

OGC (2011b), Observations and Measurements - XML Implementation, O\&M v2.0. O\&M 2.0. http://www.opengeospatial.org/standards/is, The Open Geospatial Consortium (OGC).

OGC (2011c), OGC Sensor Planning Service Implementation Standard. http://www.opengeospatial.org/standards/sps; http://www.opengis.net/doc/IS/SPS/2.0, The Open Geospatial Consortium (OGC).

STRAuss, P. \& StAudinger, B. (2007), Estimating of phosphorus and sediment loads from two main tributaries of lake Mondsee. Schriftenreihe BAW, 26, 18-33.

Swierczynski, T., Lauterbach, S., Dulski, P., Delgado, J., Merz, B. \& Brauer, A. (2013), Mid- to late Holocene flood frequency changes in the northeastern Alps as recorded in varved sediments of Lake Mondsee (Upper Austria). Quaternary Science Reviews, 80 (0), 78-90. 